\title{
Critérios de Seleção de Valores Singulares em Problemas Inversos Lineares: uma Aplicação em Tomografia de Tempos de Trânsito
}

\author{
Selection Rules for Singular Values in Linear Inverse Problems: \\ an Application on Traveltime Tomography
}

\author{
José Nilson Pereira Silva \\ Instituto de Geociências - UFBA \\ Salvador, BA, Brasil - 40001-970 \\ Amin Bassrei* \\ Instituto de Física e CPGG - UFBA \\ Salvador, BA, Brasil - 40001-970
}

\begin{abstract}
Os problemas inversos da Geofísica geralmente podem ser formulados como um sistema de equações lineares. Tais problemas inversos são geralmente mal-postos, isto é, suas soluções são condicionadas pelos aspectos da existência, unicidade e estabilidade. A tomografia sísmica é uma tipo especial de problema inverso, que permite estimar uma função a partir de uma integral de linha da mesma. Na tomografia de tempos de trânsito, objeto deste trabalho, a incógnita do problema, ou seja, o vetor de parâmetro de modelo, é a distribuição bi-dimensional de vagarosidades, que é obtida a partir do conhecimento dos tempos de trânsito entre fontes e receptores, que vem a formar o vetor de parâmetro de dado. Utilizando dados sintéticos fizemos várias simulações numa aplicação de tomografia de tempos de trânsito de transmissão. O exemplo, de médio porte, é uma idealização de um reservatório de hidrocarbonetos. Foi incorporado ruído gaussiano aos tempos de trânsito em diferentes níveis. Os resultados foram satisfatórios, gerando tomogramas bastante semelhantes aos valores verdadeiros.
\end{abstract}

Palavras-chaves: Problemas Inversos, Sismologia de Exploração, Tomografia de Tempos de Trânsito, Decomposição por Valores Singulares.

The inverse problems of Geophysics, in a general way, can be formulated as a system of linear equations. These inverse problems, in the whole, are bad formulated, that is, their solutions must satisfy the existence, uniqueness and stability conditions. The seismic tomography is a special type of inverse problem that gives an estimation of a function from a line integral of that function. In the transit-time tomography, the subject of this work, the variable of the problem, that is, the parameter vector of the model is the slowness two-dimensional distributions, which is obtained from the knowledge of transit-times between sources and receptors, so establishing the parameter vector of data. By using trial data we make many simulations testing the transit-time tomography of transmission. This example, as one of medium size, is an idealization for a hydrocarbons reservoir. We merge to the transit-times gaussian noise at different levels. The results were satisfactory with tomograms very similar to the real values.

Key-words: Inverse Problems, Exploration Seismology, Transit-time Tomography, Singular Values Decomposition.

\section{INTRODUÇÃO}

*Endereço Eletrônico: bassrei@ufba.br
Tomografia é a técnica utilizada para gerar imagens de seções transversais de um objeto a partir de dados de reflexão ou de transmissão. Essa técnica foi utilizada inicialmente em imageamento médico e em testes não des- 
trutivos. No entanto, hoje mostra-se também adequada às aplicações geofísicas tanto na Geofísica da Terra Sólida quanto na Geofísica de Exploração. Nos últimos anos a geotomografia tem sido bastante utilizada na construção de imagens da subsuperfície, em particular na caracterização e no monitoramento de reservatórios. Isto se deve pelo fato da mesma se tratar de uma ferramenta que apresenta menos restrições quanto à complexidade das estruturas geológicas.

A tomografia é um tipo de problema inverso, expresso matematicamente por $\mathbf{d}=G \mathbf{m}$, onde $\mathbf{d}$ é o vetor de parâmetros de dado, $G$ é a matriz tomográfica, e $\mathbf{m}$ é o vetor de parâmetros de modelo, este último sendo a incógnita do problema. Para a solução do problema inverso via de regra precisamos obter a matriz pseudo-inversa $G^{+}$, também chamada de inversa de Moore-Penrose. Para tanto, utilizamos o método da decomposição por valores singulares, ou SVD, do inglês singular value decomposition. O SVD fornece os valores singulares da matriz $G$, geralmente apresentadas em ordem decrescente. Os valores singulares pequenos devem ser evitados, pois distorcem a solução do problema, no caso, os tomogramas estimados. O objetivo principal deste trabalho é a seleção dos valores singulares, de modo que os pequenos valores sejam excluídos do problema. Utilizamos os critérios: (1) comportamento de decaimento dos valores singulares, (2) a derivada dos valores singulares, (3) o erro entre os vetores de parâmetros de dado observado e calculado, (4) o erro entre os vetores de parâmetros de modelo verdadeiro e estimado, (5) a energia do vetor de parâmetros de modelo estimado, e (6) a entropia do vetor de parâmetros de modelo estimado, determinamos regiões ótimas, de modo a escolher o número ideal de valores singulares a serem incorporados para a construção da solução.

A idéia de se fazer esse tipo de seleção dos valores singulares foi sugerida em [1], onde foi aplicado num pequeno exemplo de tomografia de tempos de trânsito. No presente trabalho, a abordagem sugerida é aplicada num exemplo de porte médio, e também é realizado um estudo da influência do ruído. É digno de nota mencionar que existem outras alternativas de se lidar com o problema dos pequenos valores singulares. Por exemplo, ao se empregar a regularização por matrizes de derivadas, mais conhecida como regularização de Tikhonov, podem ser utilizados todos os valores singulares. Devido à regularização da matriz tomográfica, o efeito dos pequenos valores singulares será desprezível não comprometendo a qualidade da solução estimada. Inclusive, essa técnica já é consagrada na literatura geofísica. Uma aplicação de regularização em tomografia de tempos de trânsito, mesmo objeto de interesse do presente trabalho, pode ser vista em [2]. Já em [3] a regularização por matrizes de derivadas, é aplicada na tomografia geofísica de difração.

Esse artigo está dividido em seis partes. No item Inversão de Dados e SVD fazemos uma revisão de problemas inversos e do SVD. A seguir, em Traçado Cinemático de Raios, discutimos o método utilizado para a modelagem direta, que é o traçado de raios de ondas transmitidas. No item Tomografia Sísmica, apresentamos os fundamentos da tomografia sísmica, em particular tomografia de tempos de trânsito, e, os critérios de seleção listados acima são discutidos no item Critérios para a Seleção de Valores Singulares. No item Simulações em Tomografia de Tempos de Trânsito apresentamos um exemplo com dados sintéticos e os resultados das simulações, e, por fim, apresentamos as Conclusões.

\section{INVERSÃO DE DADOS E SVD}

Matematicamente classificamos os problemas inversos como problemas do tipo malposto ou seja, que não respeitam pelo menos uma das seguintes condições: (i) existência da solução; (ii) unicidade da solução; e (iii) estabilidade da solução. Os problemas inversos geofísicos lineares, ou linearizados, podem ser formulados como um sistema linear de equações [4]:

$$
\mathbf{d}=G \mathbf{m},
$$


sendo $\mathbf{d}^{T}=\left[d_{1}, d_{2}, d_{3}, \ldots, d_{M}\right]$ e $\mathbf{m}^{T}=$ $\left[m_{1}, m_{2}, m_{3}, \ldots, m_{N}\right]$. d representa o vetor coluna dos parâmetros de dados observados, $\mathbf{m}$ o vetor coluna dos parâmetros do modelo, e $G$ é uma matriz $M \times N$ de coeficientes que relaciona os vetores $\mathbf{d}$ e $\mathbf{m}$. Supondo a matriz $G$ conhecida, pode-se resolver o sistema utilizando-se uma matriz inversa, ou seja,

$$
\mathbf{m}=G^{-1} \mathbf{d} .
$$

Entretanto, uma matriz $G$ somente é inversível se for quadrada e possuir posto completo. Como este é um caso muito específico e raro em problemas geofísicos reais, tornamse necessários procedimentos de resolução para matrizes não-quadradas e/ou com posto incompleto.

Um sistema linear pode apresentar três possibilidades diferentes de solução. (1) O sistema pode ter uma única solução $\mathbf{m}^{\text {est }}$. Neste caso classificamos o sistema como determinado $(M=N)$. (2) O sistema pode apresentar infinitas soluções, podendo ser então chamado de sobredeterminado $(M>N)$. (3) O sistema pode não apresentar nenhuma solução sendo chamado então de sistema subdeterminado $(M<N)$.

O conceito de inversa generalizada foi desenvolvido inicialmente por Moore e de forma mais completa por Penrose [5]. Considere uma matriz $G$ de dimensões $M \times N$. Se: (i) $G G^{+} G=$ $G$, (ii) $G^{+} G G^{+}=G^{+}$, (iii) $\left(G G^{+}\right)^{T}=G G^{+}$, e (iv) $\left(G^{+} G\right)^{T}=G^{+} G$, então a matriz inversa de dimensões $N \times M$, denotada por $G^{+}$, será única. O modo mais comum de se calcular a pseudo-inversa $G^{+}$é usando o SVD [6].

Uma matriz retangular $G$, de dimensões $M \times N$, com posto $k$, pode ser decomposta como:

$$
G=U \Sigma V^{T}
$$

onde $U$ é uma matriz $M \times M$ que contêm os autovetores ortonormalizados de $G G^{T}, V$ é uma matriz $N \times N$ que contêm os autovetores ortonormalizados de $G^{T} G$, e $\Sigma$ é uma matriz diagonal, $M \times N$, que contêm os valores singulares $(\sigma)$ de $G$, dispostos em ordem decrescente, isto é, $\sigma_{1} \geq \sigma_{2} \geq \ldots \geq \sigma_{k}$.

A inversa generalizada é expressa por

$$
G^{+}=V \Sigma^{+} U^{T}
$$

onde $\Sigma^{+}$é uma matriz diagomal $N \times M$, que contêm os recíprocos dos valores singulares não-nulos de $G$, isto é,

$$
\Sigma^{+}=\left(\begin{array}{cccc}
E & 0 & \cdots & 0 \\
0 & 0 & \cdots & 0 \\
\vdots & \vdots & \ddots & \vdots \\
0 & 0 & \cdots & 0
\end{array}\right)
$$

e $E$ é a matriz quadrada diagonal de ordem $k$ expressa por

$$
E=\left(\begin{array}{cccc}
\sigma_{1}^{-1} & 0 & \cdots & 0 \\
0 & \sigma_{2}^{-1} & \cdots & 0 \\
\vdots & \vdots & \ddots & \vdots \\
0 & 0 & \cdots & \sigma_{k}^{-1}
\end{array}\right)
$$

Se a solução estimada é definida por $\mathbf{m}^{\text {est }}=$ $G^{+} \mathbf{d}^{\text {obs }}$, então ela pode ser calculada pela relação:

$$
\mathbf{m}^{e s t}=V \Sigma^{+} U^{T} \mathbf{d}^{o b s}
$$

O vetor de parâmetros de modelo $\mathbf{m}$ pode ser denotado por $\mathbf{m}^{\text {ver }}$, se este for o vetor verdadeiro, ou por $\mathbf{m}^{\text {est }}$, no caso do vetor estimado, isto é, o resultado de um processo de inversão de dados. O vetor $\mathbf{m}^{\text {ver }}$ é conhecido apenas em exemplos artificiais, geralmente chamados de exemplos com dados sintéticos, uma vez que na vida real nunca se conhece $\mathbf{m}^{\text {ver }}$. Isso se deve ao fato que, em geral, não existe acesso direto ao interior do planeta, nem mesmo em profundidades mais rasas. Em exemplos sintéticos é comum calcular o erro entre $\mathbf{m}^{\text {ver }}$ e $\mathbf{m}^{\text {est }}$ tal qual apresentado no item Critérios para a Seleção de Valores Singulares.

Com o conhecimento $\mathbf{m}^{\text {est }}$ pode calcular os parâmetros de dados calculados, pela expressão:

$$
\mathbf{d}^{\text {calc }}=G \mathbf{m}^{\text {est }}=U \Sigma V^{T} \mathbf{m}^{\text {est }} .
$$

Novamente, no item Critérios para a Seleção de Valores Singulares mostramos 
como calcular o erro entre $\mathbf{d}^{\text {obs }}$ e $\mathbf{d}^{\text {calc }}$. Esse erro pode ser calculado sempre, seja em exemplos com dados sintéticos, como também quando se utiliza dados reais.

\section{TRAÇADO CINEMÁTICO DE RAIOS}

Vários métodos estão presentes na literatura para descreverem o caminho percorrido pelo raio entre dois pontos. Dentro do universo das técnicas de modelamento, o traçado de raio é uma das que mais se destacam. A sua principal utilização é na geração de sismogramas e secções sísmicas a partir de um modelo sintético que simula estruturas geológicas. A seguir descrevemos o algoritmo numérico de segunda ordem de Anderson \& Kak [7], próprio para utilização em tomografia de tempo de trânsito podendo este ser utilizado tanto na simulação de problemas diretos, como também em processos de inversão.

Seja a equação de Euler para o raio na sua forma diferencial:

$$
\frac{d}{d s}\left(n \frac{d \mathbf{r}}{d s}\right)=\nabla n,
$$

sendo o índice de refração $n$ dado por

$$
n(x, z)=\frac{c}{v(x, z)},
$$

onde $c$ é a velocidade do som no meio de referência, $v(x, z)$ é a velocidade da onda no meio, $\mathbf{r}$ é o vetor posição do raio, $d \mathbf{r} / d s$ é o vetor unitário tangente ao raio em $(x, z)$, e $d s$ é o elemento de raio. Desenvolvendo a equação de Euler obtém-se:

$$
\frac{d n}{d s} \frac{d \mathbf{r}}{d s}+n \frac{d^{2} \mathbf{r}}{d s^{2}}=\nabla n,
$$

mas,

$$
\frac{d n}{d s}=\frac{d n}{d \mathbf{r}} \frac{d \mathbf{r}}{d s}=\nabla n \cdot \frac{d \mathbf{r}}{d s},
$$

de forma que

$$
\left(\nabla n \cdot \frac{d \mathbf{r}}{d s}\right) \frac{d \mathbf{r}}{d s}+n \frac{d^{2} \mathbf{r}}{d s^{2}}=\nabla n
$$

Ao desenvolvermos o vetor posição por uma série de Taylor no ponto $(s+\Delta s)$, onde $\Delta s$ é o incremento do raio, e, levando em conta apenas os três primeiros termos da série temos que:

$$
\mathbf{r}(s+\Delta s)=\mathbf{r}(s)+\frac{d \mathbf{r}}{d s} \Delta s+\frac{1}{2} \frac{d^{2} \mathbf{r}}{d s^{2}} \Delta s^{2} .
$$

Isolando-se o vetor curvatura $\left(d^{2} \mathbf{r} / d s^{2}\right)$ na equação (1) e substituindo em (2), é obtida a seguinte aproximação para a equação do raio:

$$
\begin{aligned}
\mathbf{r}(s+\Delta s) & =\mathbf{r}(s)+\frac{d \mathbf{r}}{d s} \Delta s \\
& +\frac{1}{2 n}\left[\nabla n-\left(\nabla n \cdot \frac{d \mathbf{r}}{d s}\right) \frac{d \mathbf{r}}{d s}\right] \Delta s^{2} .
\end{aligned}
$$

Considerando dois pontos quaisquer do raio $P_{1}\left(x_{k}, z_{k}\right)$ e $P_{2}\left(x_{k+1}, z_{k+1}\right)$ separados por $\Delta s$, o vetor unitário na direção de propagação pode ser escrito como:

$$
\frac{d \mathbf{r}}{d s}=\cos \alpha_{k} \hat{\mathbf{i}}+\operatorname{sen} \alpha_{k} \hat{\mathbf{k}},
$$

onde $\hat{\mathbf{i}}$ e $\hat{\mathbf{k}}$ são, respectivamente, os vetores unitários nas direções $x$ e $z$, e $\alpha$ é o ângulo formado entre a direção do raio e o eixo horizontal. $\mathrm{O}$ ângulo $\alpha$ está associado às coordenadas pelas expressões:

$$
\text { sen } \alpha_{k+1}=\frac{z_{k+1}-z_{k}}{\Delta s},
$$

e

$$
\cos \alpha_{k+1}=\frac{x_{k+1}-x_{k}}{\Delta s} .
$$

O gradiente do índice de refração é definido como:

$$
\nabla n=\frac{\partial n}{\partial x} \hat{\mathbf{i}}+\frac{\partial n}{\partial z} \hat{\mathbf{k}}
$$

e o produto interno que aparece na equação (3) pode ser escrito da seguinte forma:

$$
\nabla n \cdot \frac{d \mathbf{r}}{d s}=n_{x} \cos \alpha_{k}+n_{z} \operatorname{sen} \alpha_{k},
$$

onde as derivadas direcionais são expressas por:

$$
n_{x}=\frac{\partial n}{\partial x},
$$


e

$$
n_{z}=\frac{\partial n}{\partial z}
$$

Considerando o meio já discretizado, as derivadas direcionais $n_{x}$ e $n_{z}$ podem ser calculadas usando diferenças finitas da seguinte forma:

$$
n_{x}(i, j)=\frac{n(i+1, j)-n(i-1, j)}{2 \Delta x},
$$

e

$$
n_{z}(i, j)=\frac{n(i, j+1)-n(i, j-1)}{2 \Delta z}
$$

O ponto seguinte do raio é calculado fazendo uso da equações acima, de modo que:

$x_{k+1}=x_{k}+\cos \alpha_{k} \Delta s+\frac{1}{2 n_{k}}\left(n_{x k}-d_{k} \cos \alpha_{k}\right) \Delta s^{2}$, e

$z_{k+1}=z_{k}+\operatorname{sen} \alpha_{k} \Delta s+\frac{1}{2 n_{k}}\left(n_{z k}-d_{k} \operatorname{sen} \alpha_{k}\right) \Delta s^{2}$,

onde $n_{k}$ representa o índice de refração, $n_{x k}$, e $n_{z k}$ as suas derivadas, e $d_{k}$ é o componente da derivada direcional no ponto $\left(x_{k}, z_{k}\right)$.

Podemos observar nessa equação que, se $n_{k}$ for substituído por $c v_{k}$, onde $v_{k}$ representa a vagarosidade do meio, a velocidade $c$ desaparece e as expressões ficam:

$x_{k+1}=x_{k}+\cos \alpha_{k} \Delta s+\frac{1}{2 v_{k}}\left(v_{x k}-d_{k} \cos \alpha_{k}\right) \Delta s^{2}$,

$\mathrm{e}$

$z_{k+1}=z_{k}+\operatorname{sen} \alpha_{k} \Delta s+\frac{1}{2 v_{k}}\left(v_{z k}-d_{k} \operatorname{sen} \alpha_{k}\right) \Delta s^{2}$,

sendo

$$
d_{k}=v_{x k} \cos \alpha_{k}+v_{z k} \text { sen } \alpha_{k}
$$

As equações (5) e (6) permitem o cálculo do traçado do raio entre fontes e receptores. Neste trabalho foi utilizado o programa FORTRAN de traçado de raios desenvolvido por Schots [8].

\section{TOMOGRAFIA SÍSMICA}

A Geofísica de Exploração tem como um dos seus principais objetivos a obtenção de imagens precisas da subsuperfície. Para tal, diversas técnicas foram introduzidas na indústria do petróleo no decorrer das últimas décadas. A geotomografia é uma dessas técnicas mais recentes. Historicamente o objetivo principal da Sismologia de Exploração é a determinação das velocidades de propagação das ondas sísmicas em subsuperfície. A tomografia sísmica é uma técnica de reconstrução de imagens a partir das somas dos valores das propriedades em determinadas direções $[9,10]$. A reconstrução tomográfica é um tipo especial de problema inverso que permite estimar uma função a partir de integrais de linha da mesma. A tomografia surgiu primeiramente na Medicina para o imageamento do corpo humano. Um conjunto de fontes e receptores gira em torno do paciente de modo a se ter uma varredura completa, o que irá proporcionar imagens de mais alta resolução. Geralmente na Medicina se utiliza raios-X. Em Geofísica, diferentemente da Medicina, não se pode realizar uma volta completa em torno do objeto de estudo. Ao invés de raios-X se utilizam ondas mecânicas ou outras ondas eletromagnéticas. A tomografia sísmica é utilizada na exploração de hidrocarbonetos para a estimação das propriedades físicas da subsuperfície, particularmente a velocidade. Existem duas classes gerais de tomografia sísmica: a tomografia de difração que é uma abordagem dinâmica, e a tomografia de tempo de trânsito, que é uma abordagem cinemática. $\mathrm{O}$ presente trabalho lida com a tomografia de tempo de trânsito, que é assim chamada porque utiliza o tempo de trânsito entre fontes e receptores. Esses tempos de trânsito constituem o chamado vetor de parâmetros de dado d, que é a informação de entrada na inverão tomográfica. A matriz $G$ utilizada na tomografia descreve a geometria dos raios que conectam fontes e receptores. A informação de saída inversão tomográfica é o vetor de parâmetros de modelo, m, que contém os valores de vagarosidade do 
meio geológico que supostamente tem um reservatório de hidrocarbonetos.

O tempo de trânsito é a integral de linha da vagarosidade ao longo do raio:

$$
t=\int_{r} s(x, z) d l
$$

onde $t$ é o tempo de trânsito, $r$ é a trajetória do raio ao longo do qual é realizada a integração, $d l$ é o elemento de raio, e $s(x, z)$ é a vagarosidade do meio no ponto $(x, z)$, onde $x$ e $z$ representam, respectivamente, as coordenadas horizontal e vertical.

Como o caminho do raio depende da vagarosidade, a equação do tempo de trânsito é não-linear. Para simplificar a resolução do problema, devemos efetuar a linearização, o que é feito pela expansão do tempo de trânsito, $\mathbf{t}_{r}=g[\mathbf{s}(x, z)]$, por uma série de Taylor em torno do ponto $\mathbf{s}^{0}$, que após a truncagem dos termos de derivada superior à primeira, resulta na expressão:

$$
\mathbf{t}=\mathbf{t}^{0}+\left.\frac{\partial g}{\partial \mathbf{s}}\right|_{\mathbf{s}=\mathbf{s}^{0}}\left(\mathbf{s}-\mathbf{s}^{0}\right) .
$$

Numa forma mais compacta a expressão acima pode ser escrita como

$$
\Delta \mathbf{t}=G \Delta \mathbf{s}
$$

Num processo iterativo, atualizado da $k$-ésima para a $(k+1)$-ésima iteração, o vetor $\Delta \mathbf{t}$ corresponde às diferenças entre o tempo de trânsito observado $\mathbf{t}^{\text {obs }}$ e o tempo de trânsito calculado $\mathbf{t}^{\text {calc }, k}$ referente à vagarosidade estimada da $k$ ésima $\mathbf{s}^{e s t, k}$. A matriz $G$ contém os elementos $g_{i j}$ que correspondem às distâncias que 0 $j$-ésimo raio percorre no $i$-ésimo bloco. $\mathrm{O}$ vetor $\Delta \mathbf{s}$ corresponde às diferenças entre as vagarosidades estimadas $\mathbf{s}^{e s t, k+1} \mathrm{e}$ as vagarosidades estimadas $\mathbf{s}^{e s t, k}$. Dessa forma a partir da última equação, podemos obter a seguinte expressão que fornece o vetor atualizado das vagarosidades estimadas, num problema tomográfico linearizado:

$$
\mathbf{s}^{e s t, k+1}=\mathbf{s}^{e s t, k}+G^{+}\left[\mathbf{t}^{\text {obs }}-\mathbf{t}^{c a l c, k}\left(\mathbf{s}^{e s t, k}\right)\right],
$$

onde $G^{+}$representa a pseudo-inversa da ma- triz $G$, para um dado traçado de raios. Para um segundo traçado de raios a matriz $G$ será atualizada, e uma nova pseudo-inversa deverá ser calculada, e assim sucessivamente.

As equações que descrevem a tomografia de tempos de trânsito, aproximadas por um modelo linear, podem então ser formuladas como $\mathbf{d}=G \mathbf{m}$, o que numa notação mais específica seria $\mathbf{t}=G \mathbf{s}$, onde $\mathbf{t}$ é o vetor de tempos de trânsito, $G$ é a matriz que descreve a geometria dos raios, e s é o vetor de vagarosidades, que representam as incógnitas do problema. A vagarosidade é o recíproco da velocidade, isto é, $s_{i}=1 / v_{i}, i=1, \ldots, N$.

Neste trabalho adotamos o último caso, ou seja, o da tomografia linear, onde a geometria dos raios não depende da distribuição de velocidades. Em outras palavras, os raios são retos, e não há necessidade de se fazer mais um traçado de raios, nem tampouco de atualizar o vetor de vagarosidades estimadas. Essa aproximação é em geral válida para pequenos contrastes de velocidades, ou de vagarosidades. A nossa escolha é justificável uma vez que o objetivo principal não é obter imagens de maior resolução da subsuperfície, e sim validar um procedimento de seleção de valores singulares.

A Figura 1 ilustra um modelo hipotético para a geometria de aquisição poço-a-poço, mostrando a disposição de fontes e receptores, assim como os caminhos de alguns raios, que conectam fontes e receptores. As fontes, representadas por estrelas, estão dispostas no poço à esquerda, e os receptores, representados por triângulos, estão dispostos no poço à direita.

\section{CRITÉRIOS PARA A SELEÇÃO DE VALORES SINGULARES}

Conforme afirmado na Introdução o emprego da técnica SVD está, via de regra, associada à existência dos pequenos valores singulares, que quando utilizados na construção da matriz inversa, implicam do comprometimento da qualidade da solução. Quando os valores sigulares são dispostos em ordem descrescente existe um valor singular limite, de modo 
que se o próximo valor singular for utilizado irá comprometer a solução. Por vezes não é possível determinar esse ponto ótimo. Nestes casos o melhor que se pode fazer é estabelecer uma região, não de um único ponto e sim de vários ou até muitos pontos. Para a determinação desse ponto ótimo ou dessa região ótima, propomos a utilização dos critérios a seguir.

Amplitude dos valores singulares. O programa de SVD fornece os valores singulares em ordem decrescente, isto é, à medida que o índice vai aumentando a amplitude do valor singular vai diminuindo. Os valores singulares agregam informação que é importante para a contrução da matriz pseudo-inversa. No entanto, a partir de certo valor, essa informação passa a ser considerado ruído e compromete a qualidade da solução estimada. Note-se a matriz pseudo-inversa é construída a partir dos valores de $1 / \sigma$ e não de $\sigma$.

Derivada do valor singular. A derivada de uma função $f(x)$ em um determinado ponto $x_{1}$ denotada por $f^{\prime}\left(x_{1}\right)$, é definida pelo limite:

$$
f^{\prime}\left(x_{i}\right)=\lim _{x_{i+1} \rightarrow x_{i}} \frac{f\left(x_{i+1}\right)-f\left(x_{i}\right)}{x_{i+1}-x_{i}},
$$

onde $f\left(x_{i}\right)$ e $f\left(x_{i+1}\right)$ são, respectivamente, os valores da amplitude do valor singular em um ponto e o seu subseqüente. É importante salientar que, como trata-se de uma curva crescente e negativa, os gráficos a serem apresentados nas figuras foram gerados usando-se o módulo da derivada.

Erro do dado. A partir da análise dos resultados obtidos na inversão tomográfica tornase necessário a quantificação da diferença entre os tempos de trânsito observados e os calculados. Esse erro é expresso como:

$$
\epsilon_{d}=\frac{\sqrt{\sum_{i=1}^{M}\left(t_{i}^{o b s}-t_{i}^{c a l c}\right)^{2}}}{M},
$$

onde $M$ é o número de raios (número de fontes $\times$ número de receptores), $t_{i}^{\text {obs }}$ são os tempos de trânsito observados, e $t_{i}^{\text {calc }}$ são os tempos de trânsito calculados.
Se utilizado em termos de porcentagem, o erro relativo é representado por:

$$
\epsilon_{d}(\%)=\frac{\sqrt{\sum_{i=1}^{M}\left(t_{i}^{\text {obs }}-t_{i}^{\text {calc }}\right)^{2}}}{\sqrt{\sum_{i=1}^{M}\left(t_{i}^{o b s}\right)^{2}}} \times 100 .
$$

Erro do modelo. Definimos o erro do modelo como a quantificação da diferença entre as vagarosidades consideradas como verdadeiras e aquelas estimadas para o modelo. $\mathrm{O}$ erro do modelo pode ser descrito pela expressão:

$$
\epsilon_{m}=\frac{\sqrt{\sum_{i=1}^{N}\left(s_{i}^{v e r}-s_{i}^{e s t}\right)^{2}}}{N},
$$

onde $N$ é o número de blocos da malha, $s_{i}^{\text {ver }}$ são as vagarosidades verdadeiras, e $s_{i}^{\text {est }}$ são as vagarosidades estimadas.

Se utilizado em termos de porcentagem, o erro relativo é representado por:

$$
\epsilon_{m}(\%)=\frac{\sqrt{\sum_{i=1}^{N}\left(s_{i}^{v e r}-s_{i}^{e s t}\right)^{2}}}{\sqrt{\sum_{i=1}^{N}\left(s_{i}^{v e r}\right)^{2}}} \times 100 .
$$

Energia. Definimos por energia do modelo o produto escalar do vetor de vagarosidades estimadas por ele mesmo, ou seja:

$$
E=\sum_{i=1}^{N}\left(s_{i}^{e s t} \cdot s_{i}^{e s t}\right) .
$$

O valor da energia mede o quão estável é a solução. Quanto menor for a sua variação em um determinado intervalo, mais confiáveis são os resultados obtidos para o mesmo.

Entropia. Definimos como entropia do modelo o somatório do produto entre a vagarosidade estimada e o logaritmo do inverso 
da mesma conforme podemos ver na expressão:

$$
H=\sum_{i=1}^{N} s_{i}^{e s t} \cdot \log \frac{1}{s_{i}^{e s t}} .
$$

Pela Teoria da Informação entropia representa a informação média de um processo. Neste trabalho soluções mais suaves estariam associadas a uma menor entropia.

Adição de Ruído. Foram introduzidos diferentes níveis de ruídos para verificar como se comportariam as curvas que são geradas em função das vagarosidades. A equação que define a incorporação do ruído é expressa por

$$
t_{j}^{r}=t_{j}^{o b s}+\alpha r_{j} t_{j}^{o b s}, j=1, \ldots, M,
$$

onde $t_{j}^{r}$ é o tempo de trânsito corrompido com ruído gaussiano, $t_{j}^{o b s}$ é o tempo de trânsito livre de ruído, $\alpha$ é o fator de ruído, e $r_{j}$ uma é a seqüência de números aleatórios na faixa $[-0.5,0.5]$. Foram introduzidos nas simulações cinco diferentes níveis de ruído, conforme os seguintes valores de $\alpha: 0.001 ; 0.005 ; 0.01 ; 0.05$; 0.1 ; além de uma simulação sem ruído, isto é, com $\alpha=0$. É digno de nota que o fator de ruído $\alpha$ não deve ser confundido com o ângulo do traçado de raios, também denotado por $\alpha$, conforme apresentado no item Traçado Cinemático de Raios.

\section{SIMULAÇÕES EM TOMOGRAFIA DE TEMPOS DE TRÂNSITO}

O modelo adotado é um meio geológico discretizado em 900 blocos, com 30 blocos na horizontal e 30 na vertical, conforme pode ser visto na Figura 2. Cada bloco tem o formato de um quadrado com $30 \mathrm{~m}$ de aresta. As principais feições são uma camada de baixa velocidade, com $1.700 \mathrm{~m} / \mathrm{s}$ e uma heterogeneidade de alta velocidade, com $3.400 \mathrm{~m} / \mathrm{s}$, que seria o reservatório com hidrocarbonetos. A tomografia deve fornecer a velocidade estimada de cada bloco, que modo que seriam então 900 incógnitas. A aquisição de dados é do tipo poço-a-poço, onde 30 fontes estão distribuídas com espaçamento uniforme no poço da esquerda, e 30 receptores, também com espaçamento regular no poço da direita. Dessa forma, o número de raios, ou o número de equações, ou ainda o número de informações, é 900 .

A Figura 3 mostra a curva decrescente dos valores singulares, onde podemos observar que os valores singulares com índice acima de 500 são muito pequenos. Uma análise nos valores permite concluir que o valor singular é virtualmente nulo em algum índice entre 800 e 900, de modo que será dada ênfase para esta faixa.

A Figura 4(a) mostra os valores singulares entre 800 e 900, onde pode se observar uma acentuada queda. Aplicando a escala logarítmica no eixo vertical obtivemos a curva mostrada na Figura 4(b). A derivada da curva da amplitude dos valores singulares é mostrada na Figura 4(c). Pela inspeção visual das Figuras 4(b) e 4(c) concluimos que o ponto crítico é entre 800 e 830, isto é, a inclusão de valores singulares com índice acima desse ponto irá comprometer a qualidade da solução.

A aplicação do critério do erro dos parâmetros de dados pode ser visto na Figura 5 (a), onde se apresenta o valor de $\epsilon_{d}$ em função do número de valores singulares, para diferentes níveis de ruído. O nível de ruído é dado pelo parametro $\alpha$, que assumiu os valores de 0 (sem ruído); 0.001; 0.005; 0.01; 0.05 e 0.1. O gráfico começa em 800 , uma vez que se constatou que o ponto crítico é acima de 800 . Por questão de limitação de espaço o gráfico completo, ou seja, de 1 a 900 valores singulares não está sendo mostrado. $\mathrm{O}$ fato do erro $\epsilon_{d}$ aumentar quando se aumenta o nível de ruído é coerente. Há uma superposição das curvas para os valores de $\alpha 0 ; 0.001$ e 0.005 . Detalhando mais a escala vertical verificamos que não ocorreu mais a superposição, conforme pode ser visto na Figura 5 (b). Entre as três curvas, há novamente uma coerência entre o erro $\epsilon_{d}$ e o nível de ruído. Pela Figura 5(b) constatamos que o valor de $\epsilon_{d}$ é estável até aproximadamente 830 valores singulares, e depois apresenta uma tendência crescente. Portanto o número máximo de valores singulares a ser utilizado na inversão deve ser algo menor que 830 ou 835. Essa observação é coerente com as conlcusões obtidas a partir 
das Figuras 4(b) e 4(c).

O resultado do critério do erro dos parâmetros de modelo (não mostrado) indicou que o valor de $\epsilon_{m}$ é estável até aproximadamente 830 ou 835 valores singulares, e depois o mesmo apresenta uma tendência crescente. Essa observação é idêntica ao que ocorreu em relação do critério de $\epsilon_{d}$, ou seja, o número máximo de valores singulares a ser utilizado na inversão deve ser algo menor que 830 ou 835 . Esse critério é o único que não pode ser utilizado em dados reais, uma que não se conhece o valor do vetor de vagarosidades verdadeiras $\left(\mathbf{s}^{v e r}\right)$. Neste trabalho, a utilização do critério de $\epsilon_{m}$ é justificada porque as simulações lidam com um conjunto de dados sintéticos, ou seja, existe um conhecimento prévio do vetor $\mathbf{s}^{v e r}$. Além disso, deseja-se comparar ou até validar outros critérios com o do $\epsilon_{m}$.

A aplicação do critério da energia $E$ dos parâmetros de modelo pode ser visto na Figura 6(a), onde se apresenta o valor de $E$ em função do número de valores singulares, para os mesmos níveis de ruído usados acima. Para o caso específico da faixa entre 800 e 830 valores singulares, podemos afirmar que o fato das curvas para $\alpha=0.01, \alpha=0.05$ e $\alpha=0.1$ não se apresentarem sobrepostas, permite concluir que a energia $E$ é maior, quanto maior for o fator $\alpha$. Todas as curvas apresentam uma abrupta variação de $E$ em torno de 830 valores singulares, havendo uma total superposição. A Figura 6(b) apresenta as mesmas curvas com uma mudança na escala vertical, onde observamos uma nítida separação entre as seis curvas, na faixa entre 830 a 860 valores singulares. Note-se que, diferentemente das figuras para os dois critérios anteriores, efetuamos aqui uma redução da escala. O critério da energia $E$ sugere que as soluções são estáveis até cerca de 830 ou 835 valores singulares, quando então começa uma súbita instabilidade, implicando em soluções sem nenhum fundamento físico. Essa observação reforça as conclusões obtidas a partir das curvas de $\epsilon_{d}$ e $\epsilon_{m}$.

O critério da entropia dos parâmetros de modelo pode ser visto na Figura 7(a), onde se apresenta o valor de $H$ em função do número de valores singulares, para os mesmos níveis de ruído. A Figura 7(b) apresenta as mesmas curvas num detalhe maior. As duas figuras indicam que a entropia aumenta à medida que o nível de ruído é maior, fato semelhante com o que ocorre com os critérios de $\epsilon_{d}, \epsilon_{m}$ e $E$. Também, ambas figuras sugerem que a entropia é estável até aproximadamente 830 valores singulares, e depois apresenta uma tendência crescente. Valores grandes de entropia representam soluções mais complexas, o que significa aqui soluções com muitas flutuações, distantes da realidade física. Mais uma vez concluimos que o número máximo de valores singulares a ser utilizado na inversão deve ser em torno de 830 ou 835.

A conjugação dos critérios aplicados permite concluir que o número ideal de valores singulares, para este problema, é em torno de 830 ou 835. Escolhendo, por exemplo, 835 valores singulares, obtivemos o tomograma da Figura 8 que indica as velocidades estimadas, no caso para $\alpha=0$. Note-se a grande semelhança entre esse resultado e a Figura 2 que mostra o tomograma verdadeiro. Note-se que essa abordagem poderia ser mais apurada se a escala horizontal fosse de maior detalhe, como por exemplo, entre 820 e 840 valores, ao invés de 800 a 900 valores singulares. Além disso, restrigindo o faixa de valores singulares, pode-se trabalhar com tabelas de números e não mais com curvas.

\section{CONCLUSÕES}

A tomografia de tempos de trânsito, é uma abordagem cinemática da tomografia de raios, que utiliza os tempos de trânsito entre fontes e receptores. A tomografia é um tipo de problema inverso, que pode ser resolvido, por exemplo, utilizando a usual técnica da decomposição por valores singulares. A presença de pequenos valores singulares perturbam os resultados, gerando um crescimento anômalo em todos os critérios que envolvem o modelo estimado, quais sejam: o erro entre os dados observados e calculados, o erro entre os parâmetros de modelo verdadeiros e estimados, a energia 
do modelo estimado e a entropia do modelo estimado. Utilizando estes quatro critérios mais o comportamento do decaimento dos valores singulares, e a derivada dos valores singulares, concluímos que, para o exemplo numérico apresentado, o número ótimo utilizado foi em torno de 830 ou 835 valores singulares, dentro de um conjunto de 900 valores. A utilização de diferentes níveis de ruído mostrou-se válida, tendo em vista que a metodologia se apresentou robusta em relação à adição de ruído, e, os valores dos critérios foram proporcionais aos diferentes níveis de ruídos. Os resultados foram satisfatórios, pois os tomogramas gerados utilizando esta metodologia, tal qual o apresen- tado, apresentaram-se bastante semelhantes ao tomograma verdadeiro.

\section{Agradecimentos}

Os autores agradecem ao LAGEP Laborotório de Geofísica de Exploração de Petróleo do CPGG/UFBA pela utilização dos recursos computacionais. A. Bassrei agradece ao CNPq pelo apoio aos projetos 484.239/20066 (Edital Universal MCT/CNPq 02/2006) e 308.875/2007-9 (Bolsa de Pesquisa).
[1] A. Bassrei, A simple procedure for the selection of singular values in acoustical travel time tomography (abstract). J. Acoust. Soc. Am. 108, 2593 (2000).

[2] E.T.F. Santos, A. Bassrei, J. Costa, Evaluation of L-curve and $\Theta$-curve approaches for the selection of regularization parameter in anisotropic traveltime tomography. Journal of Seismic Exploration 15, 245 (2006).

[3] E.T.F. Santos, A. Bassrei, L- and $\Theta$-curve approaches for the selection of regularization parameter in geophysical diffraction tomography. Computers \& Geosciences 33, 618 (2007).

[4] W. Menke, Geophysical Data Analysis: Discrete Inverse Theory. Orlando: Academic Press (1984).

[5] R. Penrose, A generalized inverse for matrices. Proceedings of the Cambridge Philosoph- ical Society 51, 406 (1955).

[6] C. Lanczos, Linear Differential Operators. London: Van Nostrand (1961).

[7] A.H. Anderson, A.C. Kak, Digital ray tracing in two-dimensional refractive fields. J. Acoust. Soc. Am. 72, 1593 (1982).

[8] H.A. Schots, Tomografia Sísmica Poço-a-Poço e Poço-a-Superfície Utilizando Ondas Diretas. Dissertação (Mestrado) - Instituto de Geociências da UFBA, Salvador (1990).

[9] R.R. Stewart Exploration Seismic Tomography: Fundamentals. Tulsa: Society of Exploration Geophysicists (1991).

[10] T.-w. Lo, P.L. Inderwiesen, Fundamentals of Seismic Tomography. Geophysical Monograph Series. Tulsa: Society of Exploration Geophysicists (1994). 


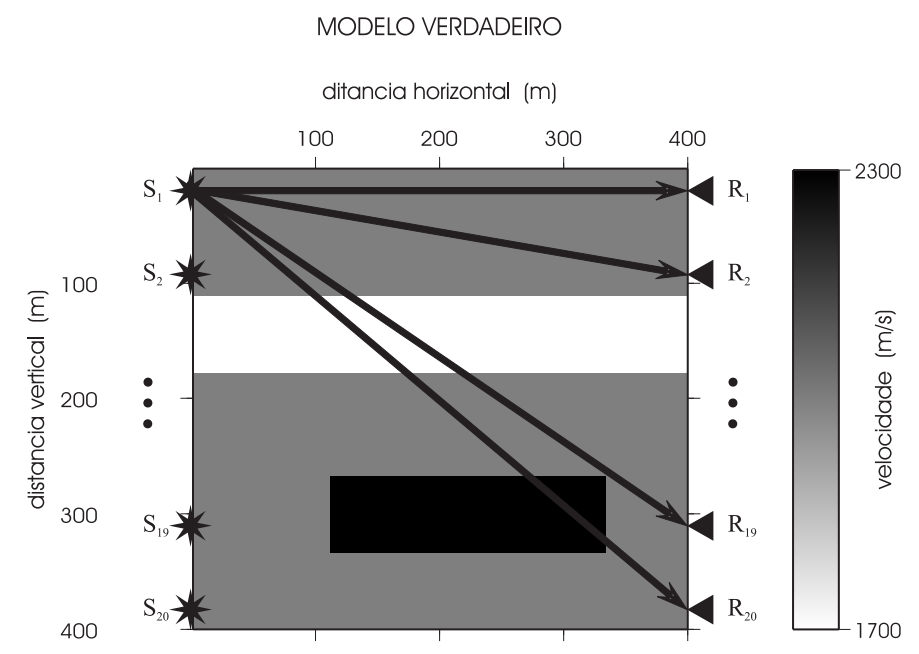

FIGURA 1 - Modelo hipotético mostrando a disposição de fontes e receptores, assim como os caminhos de alguns raios, que conectam fontes e receptores. As fontes, representadas por estrelas, estão dispostas no poço à esquerda, e os receptores, representados por triângulos, estão dispostos no poço à direita.

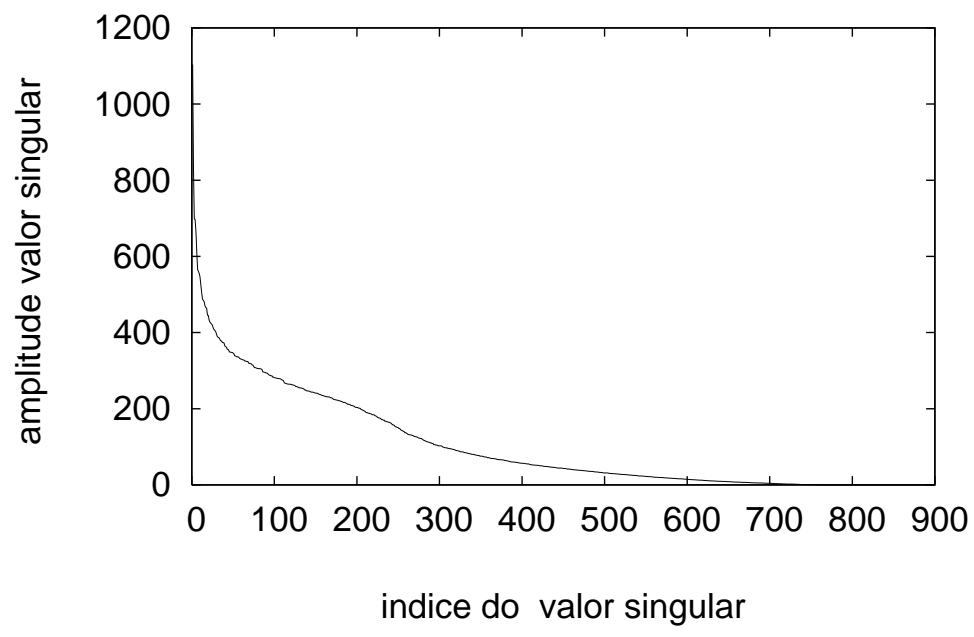

FIGURA 3 - Curva de amplitude dos valores singulares versus o índice do valor singular. Nota-se o rápido decaimento na curva, que passa a ser um caráter mais suave, se for utilizada a escala logarímica no eixo vertical. 

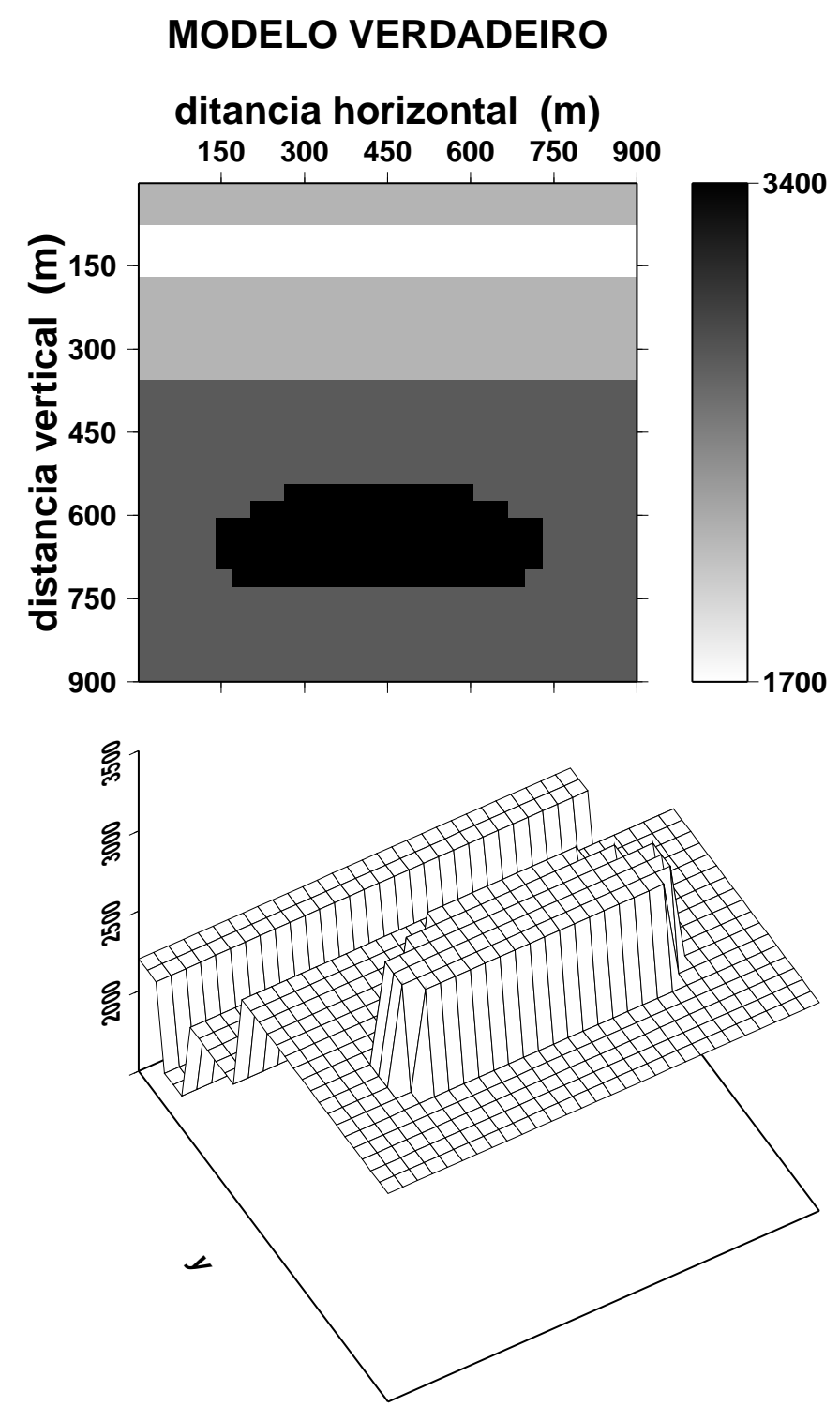

FIGURA 2 - Modelo utilizado para as simulações, com 900 blocos, sendo 30 blocos por linha horizontal e 30 blocos por linha vertical. A aquisição de dados é do tipo poço-a-poço com 30 fontes regularmente espaçados no poço à esquerda. Igual número de receptores, também regularmente espaçados, no poço à direita. 
(a)

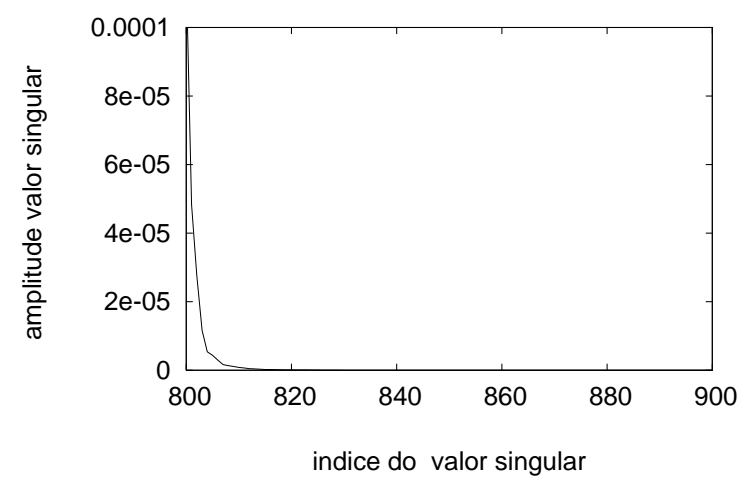

(b)

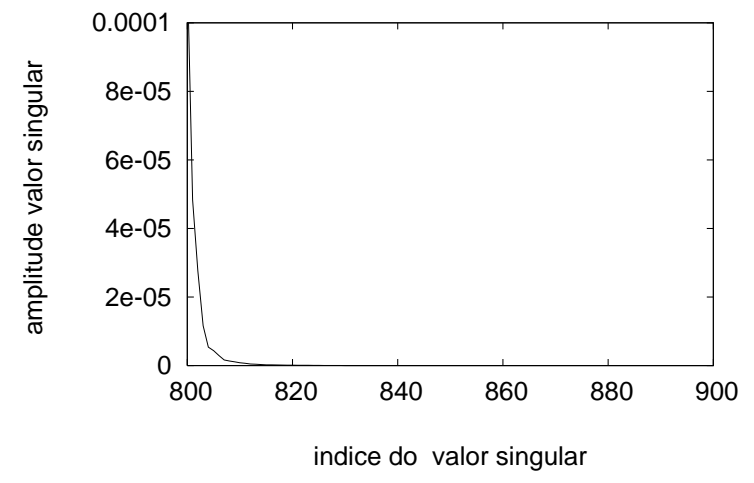

(c)

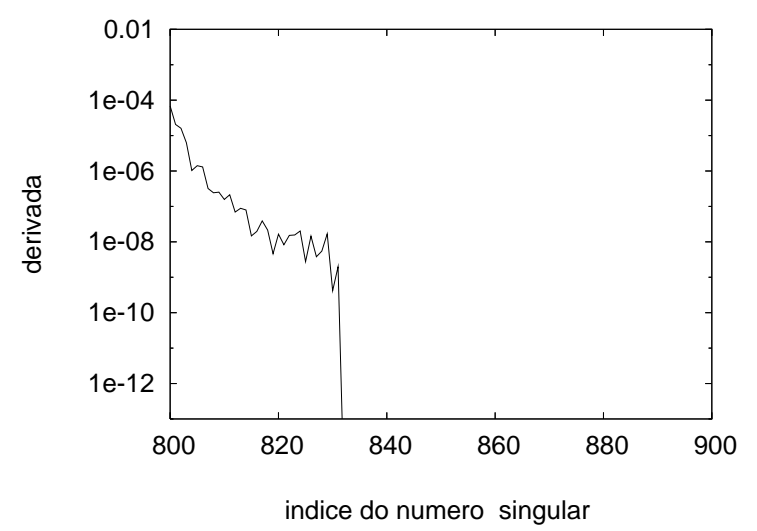

FIGURAS 4(a), 4(b) e 4(c) - Curvas restritas à faixa de 800 a 900 valores singulares, sendo: (a) curva de amplitude de valores singulares versus o índice do valor singular; (b) o mesmo do item (a) porém com escala logarítmica no eixo vertical; (c) curva do logaritmo do módulo da derivada da amplitude de valores singulares versus o índice do valor singular. 
(a)

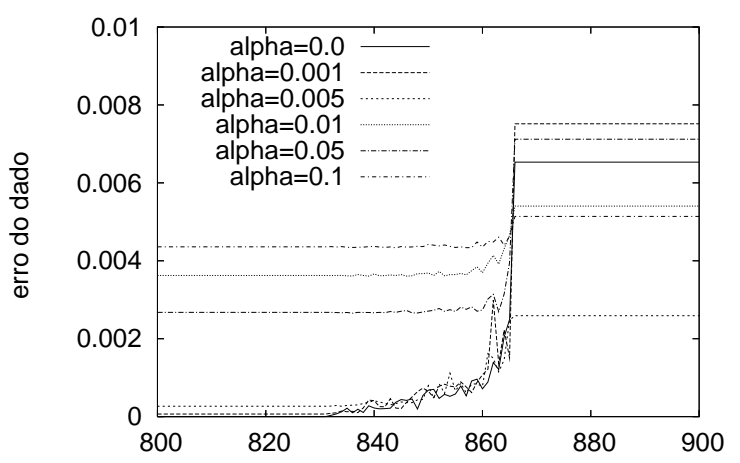

numero de valores singulares

(b)

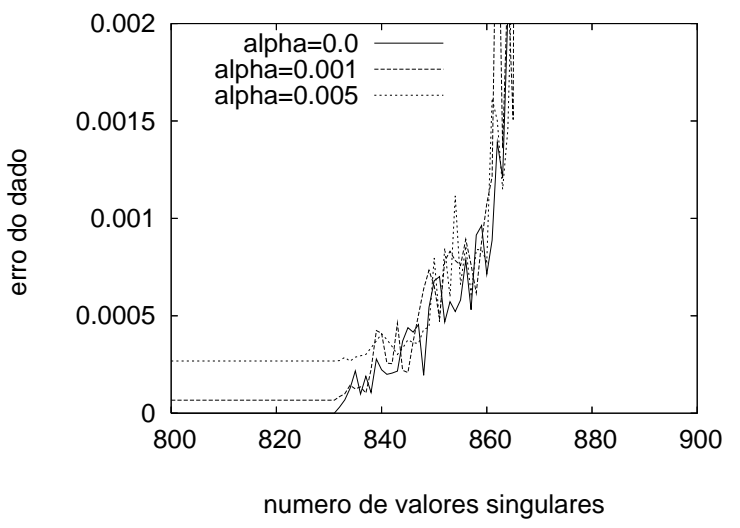

FIGURAS 5(a) e 5(b) - Curvas restritas à faixa de 800 a 900 valores singulares, para diferentes níveis de ruído, sendo: (a) curva do erro $\epsilon_{d}$ dos parâmetros de dados versus o número de valores singulares; (b) o mesmo do item (a) porém com detalhe na escala vertical, destacando as curvas para $\alpha=0, \alpha=0.001$ e $\alpha=0.005$. 
(a)

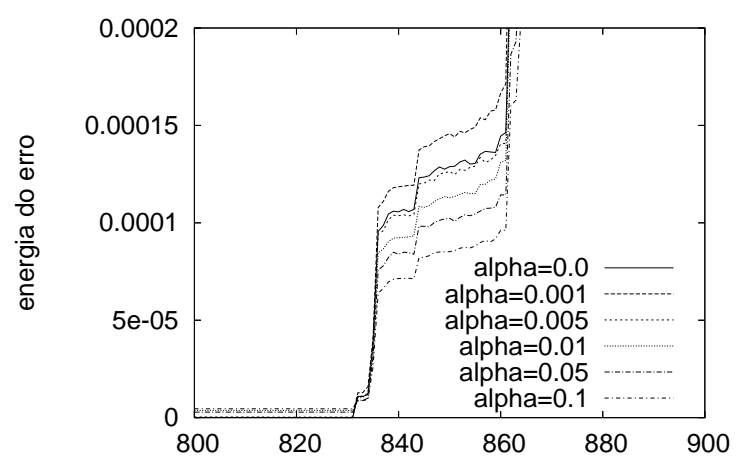

numero de valores singulares

(b)

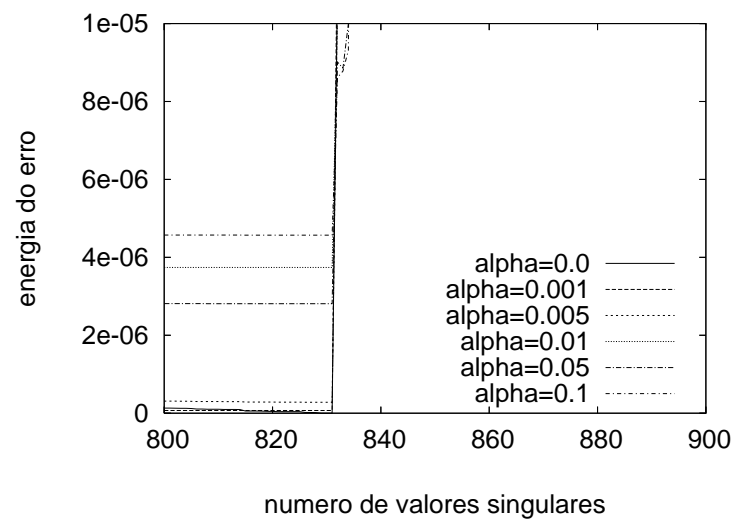

FIGURAS 6(a) e 6(b) - Curvas restritas à faixa de 800 a 900 valores singulares, para diferentes níveis de ruído, sendo: (a) curva da energia $E$ dos parâmetros de modelo versus o número de valores singulares; (b) o mesmo do item (a) porém com menos detalhe na escala vertical. Note-se que diferente das curvas para $\epsilon_{d}$ e $\epsilon_{m}$, efetuamos aqui uma redução de escala, de modo a poder visualizar uma distinção entre os diferentes níveis de ruído na faixa de 830 a 860 valores singulares. 
(a)

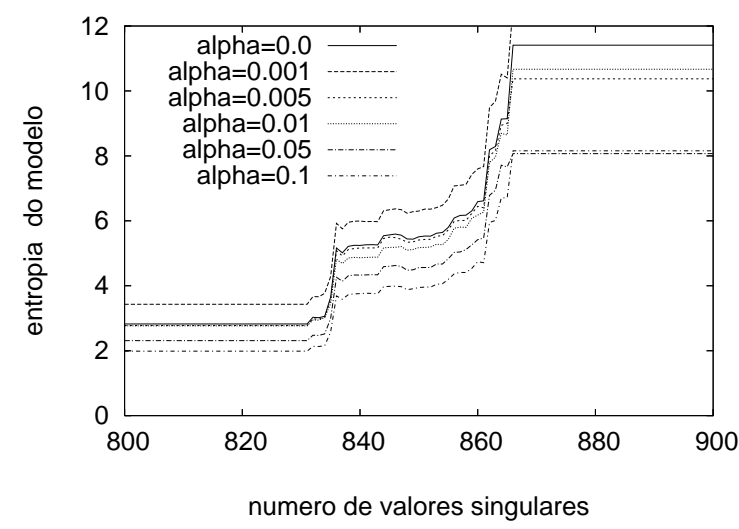

(b)

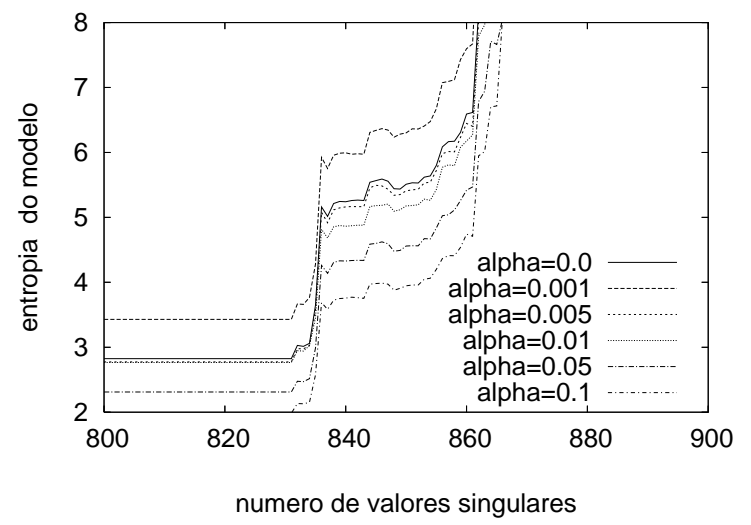

FIGURAS 7(a) e 7(b) - Curvas restritas à faixa de 800 a 900 valores singulares, para diferentes níveis de ruído, sendo: (a) curva da entropia $H$ dos parâmetros de modelo versus o número de valores singulares; (b) o mesmo do item (a) porém com detalhe na escala vertical. 

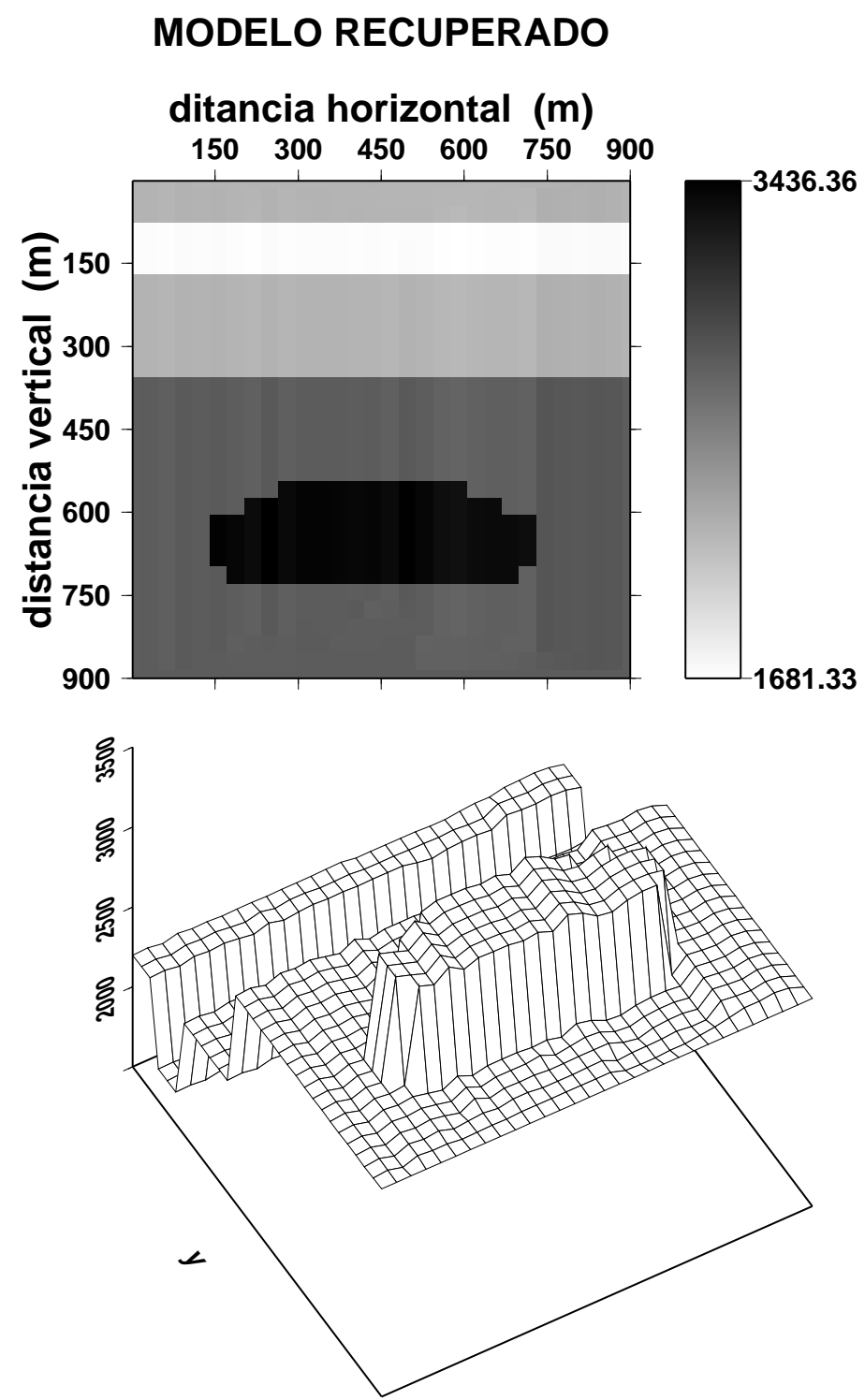

FIGURA 8 - Modelo estimado utilizando 835 valores singulares, com dados livre de ruído $(\alpha=0)$. 